\title{
Orthogonal Complex Filter Banks and Wavelets: Some Properties and Design
}

\author{
Xiao-Ping Zhang, Member, IEEE, Mita D. Desai, Senior Member, IEEE, and Ying-Ning Peng, Senior Member, IEEE
}

\begin{abstract}
Recent wavelet research has primarily focused on real-valued wavelet bases. However, complex wavelet bases offer a number of potential advantageous properties. For example, it has been recently suggested that the complex Daubechies wavelet can be made symmetric. However, these papers always imply that if the complex basis has a symmetry property, then it must exhibit linear phase as well. In this paper, we prove that a linearphase complex orthogonal wavelet does not exist. We study the implications of symmetry and linear phase for both complex and real-valued orthogonal wavelet bases. As a byproduct, we propose a method to obtain a complex orthogonal wavelet basis having the symmetry property and approximately linear phase. The numerical analysis of the phase response of various complex and real Daubechies wavelets is given. Both real and complex-symmetric orthogonal wavelet can only have symmetric amplitude spectra. It is often desired to have asymmetric amplitude spectra for processing general complex signals. Therefore, we propose a method to design general complex orthogonal perfect reconstruct filter banks (PRFB's) by a parameterization scheme. Design examples are given. It is shown that the amplitude spectra of the general complex conjugate quadrature filters (CQF's) can be asymmetric with respect the zero frequency. This method can be used to choose optimal complex orthogonal wavelet basis for processing complex signals such as in radar and sonar.
\end{abstract}

\section{INTRODUCTION}

$\mathbf{T}$ HE USE OF wavelet transforms (WT's) is becoming ubiquitous in signal processing, owing to the power of the multiresolution technique and the existence of fast algorithms [3], [4]. Research on wavelet theory is tightly connected with the filter bank work through Mallat's algorithm [5]-[7]. Both orthogonal and biorthogonal wavelet bases correspond to perfect reconstruct filter banks (PRFB's). Research on wavelets mainly concerns real-valued wavelet bases and filter banks, as is evident by a large number of publications on the subject [5]-[14]. However, complex wavelet bases and filter banks have seldom been discussed.

Manuscript received November 1, 1997; revised November 30, 1998. This work was supported in part by NASA under Grant NAG10-0155 and the Texas Advanced Research program under Grant ARP 002-1996. The associate editor coordinating the review of this paper and approving it for publication was Dr. Ahmed Tewfik.

X.-P. Zhang was with Tsinghua University, Beijing, China, and the University of Texas, San Antonio, TX 78249 USA. He is now with the Communications Research Laboratory, McMaster University, Hamilton, Ont., Canada L8S 4K1 (e-mail: zhangxp@mcmaster.ca).

M. Desai is with the Division of Engineering, University of Texas, San Antonio, TX 78249 USA.

Y.-N. Peng is with the Department of Electronic Engineering, Tsinghua University, Beijing, China.

Publisher Item Identifier S 1053-587X(99)02143-1.
Both the continuous wavelet transform (CWT) and Fourier transform are defined for signals that are complex as well as real. The basis functions of the Fourier transform are the complex exponential functions, and the FFT is equivalent to implementing a bank of bandpass complex-valued filters. Similarly, the short time Fourier transform (STFT) is equivalent to implementing a bank of bandpass complex-valued filters having equal bandwidths. The mother wavelets of the CWT are often complex-valued functions, such as the Morlet wavelet and the Gabor wavelet, etc. Present theory and techniques have already shown that these approaches are better able to handle complex signals such as in radar and sonar applications [15]-[17]. Therefore, adopting complex filter banks and associated wavelet bases for similar applications is a natural choice. For example, in radar and sonar applications, the complex I/Q orthogonal signals can be processed with complex filter banks rather than processing the I/Q channels separately. Therefore, the complex orthogonal wavelet may prove to be a good choice since it will allow us to process both the magnitude and phase simultaneously.

Recently, a few authors [1], [2], [18]-[20] have studied complex-valued filter banks. The complex Daubechies wavelet and some applications were discussed in [1], [2], [18], and [19]. In [1], the symmetric complex Daubechies wavelets were constructed. These wavelets are used for image coding applications in [2] and showed better compression performance than asymmetric wavelets. The parameterization of complex Daubechies wavelets is developed in [18]. The symmetric complex Daubechies wavelets were also used in stereo image matching in [19]. It is shown in these papers that the complex Daubechies wavelet can be symmetric, whereas the real Daubechies wavelet cannot. However, all these authors seem to be confused about the symmetry property of the filter with its linear-phase response. They always imply that if the complex basis has a symmetry property, then it must exhibit linear phase as well. Symmetry and linear phase are often critically important filter constraints. The symmetry property of the filter makes it easy to handle the boundary problem for finite length signals [14]. The linear-phase response of the filter precludes the nonlinear phase distortion and keeps the shape of the signal [21], which is usually important in image and audio signal analysis. They are both desired properties of a filter. In this paper, we prove the impossibility of a linear-phase complex orthogonal wavelet. However, a method to achieve both symmetry and approximate linear phase on a complex Daubechies wavelet is proposed. Along the way, we present 
numerical analyses of various interesting phase properties of Daubechies wavelets.

We also propose methods for designing general orthogonal complex PRFB's and give design examples. It is shown that the general complex conjugate quadrature filter (CQF) has a very flexible frequency response. The amplitude-frequency responses of the general complex CQF's can be asymmetric with respect to the zero frequency, whereas the symmetric complex CQF's and real CQF's must have symmetric amplitude-frequency responses. This property can be useful when processing complex-valued signals, such as radar and sonar I/Q orthogonal complex signals with Doppler phase shift. For example, the moving target detector (MTD) is usually a specific type of complex-valued filter bank with asymmetric amplitude spectrum. In any Doppler processing application, the positive and the negative frequencies have a different meaning. Therefore, a filter must be able to distinguish between the positive and negative frequencies. Hence, the complex filters with asymmetric amplitude spectrum promise to be very useful for processing these complex signals.

This paper is organized as follows. In Section II, compactly supported symmetric orthogonal complex-valued wavelet bases are discussed, and implications of the linear phase property are explored. Numerical analysis of the phase response of various Daubechies' orthogonal scaling functions is also presented, and the results are compared. In Section III, the design of general orthogonal complex PRFB's and associated orthogonal complex wavelets are proposed, and some examples are given. We conclude the paper in Section IV.

Notations: Some notations used in this paper are shown here. The bar or superscript "*," such as $\bar{h}$ or $h^{*}$, denotes the conjugation. $H\left(e^{j \omega}\right)$ or $\hat{h}(\omega)$ denotes the Fourier transform of discrete or continuous function $h . H(z)$ denotes the $z$ transform of discrete-time function of $h[n]$. For polynomial matrix or vector $\mathbf{H}(z), \mathbf{H}^{T}(z)$ denotes the transpose of $\mathbf{H}(z)$; $\mathbf{H}^{H}(z)$ denotes the conjugate transpose of $\mathbf{H}(z) ; \mathbf{H}_{*}(z)$ denotes the conjugation of all the coefficient of $\mathbf{H}(z)$; and $\tilde{\mathbf{H}}(z) \triangleq \mathbf{H}_{*}^{T}\left(z^{-1}\right)$. The downsampling operator $[\downarrow M]$ and upsampling operator $[\uparrow M]$ are defined as

$$
[\downarrow M] x[n] \triangleq x[M n]
$$

and

$$
[\uparrow M] x[n] \triangleq \begin{cases}x[n / M], & \text { if } n \text { is a multiple of } M \\ 0, & \text { otherwise }\end{cases}
$$

respectively.

\section{COMPACTly Supported Symmetric ORTHOGONAL COMPLEX-VALUED WAVELET BASES}

\section{A. The Symmetry of Compactly Supported Orthogonal Wavelet Bases}

Daubechies has shown that all compactly supported real orthogonal wavelet bases and their associated CQF FB's are neither symmetric nor antisymmetric, except Haar functions [22], [23]. Lawton [1] and Lina et al. [18] showed that it is possible to have the symmetry property for a complex Daubechies wavelet. In addition to their results, we show the relationship between compactly supported real orthogonal wavelet bases and compactly supported complex orthogonal wavelet bases. This is given as follows.

Proposition 1: The scaling filter $h$ in CQF PRFB associated with the dyadic compactly supported complex orthogonal wavelet basis cannot be antisymmetric.

Proof: The length $N$ of the scaling filter $h$ in CQF PRFB associated with the dyadic compactly supported orthogonal wavelet basis must be even [13], [22]. Therefore, assuming $h$ is antisymmetric, i.e., $h[n]=-h[N-1-n]$, we have $\sum_{k=0}^{N-1} h[k]=0$. In addition, it is known [3], [13], [22] that $\left|H\left(e^{j \omega}\right)\right|_{\omega=0}=\left|\sum_{k=0}^{N-1} h[k]\right|=\sqrt{2} \neq 0$. Therefore, the assumption of antisymmetry does not hold, i.e., $h$ cannot be antisymmetric.

Since the scaling function and the scaling filter have the same symmetry property [13], the associated scaling function cannot be antisymmetric either. More generally, if complex CQF PRFB's have any symmetry property, then they can only be symmetric and not antisymmetric.

Theorem 1: If there exists a symmetric complex-valued CQF PRFB, then there also exists a corresponding real-valued CQF PRFB having scaling filters with identical amplitude spectra.

Proof: According to the Daubechies method for the construction of real compactly supported orthogonal wavelet basis [22], [23], let

$$
p_{0}\left(e^{j \omega}\right)=\frac{1}{\sqrt{2}} H\left(e^{j \omega}\right)
$$

where $H$ is the scaling filter. For $p_{0}$ to have regularity $K \geq 1$, $p_{0}$ should have the form

$$
p_{0}\left(e^{j \omega}\right)=\left(\frac{1+e^{j \omega}}{2}\right)^{K} Q\left(e^{j \omega}\right)
$$

and $p_{0}$ should satisfy

$$
P_{0}\left(e^{j \omega}\right)+P_{0}\left(e^{j(\omega+\pi)}\right)=1
$$

where $P_{0}\left(e^{j \omega}\right)=\left|p_{0}\left(e^{j \omega}\right)\right|^{2}$

Given the scaling filter $H_{c}$ for any symmetric complex CQF PRFB, and since $h_{c}[n]=h_{c}[N-1-n]$, we have

$$
H_{c}\left(e^{j \omega}\right)=e^{-j \omega(N-1)} H_{c}\left(e^{-j \omega}\right)
$$

Thus

$$
\left|H_{c}\left(e^{j \omega}\right)\right|^{2}=\left|H_{c}\left(e^{-j \omega}\right)\right|^{2}
$$

i.e., its amplitude spectrum is an even function.

Because $\left|H_{c}\left(e^{j \omega}\right)\right|^{2}$ is an even function, it can be written as a real-valued polynomial of $\cos (\omega)$. Furthermore, $\left|H_{c}\left(e^{j \omega}\right)\right|^{2}$ fulfills the required conditions of CQF since $H_{c}$ is a CQF. 
Therefore, $\left|H_{c}\left(e^{j \omega}\right)\right|^{2}$ is also a solution of the Daubechies' equation (3). Then, by using the zero decomposition method of Daubechies (see [22] and [23]), we can find a scaling filter $h$ associated with a real CQF PRFB, whose amplitude spectrum is $\left|H_{c}\left(e^{j \omega}\right)\right|$.

In Theorem 1, it is shown that for every symmetric complexvalued CQF PRFB, there is a corresponding real-valued CQF PRFB. It has already been shown that the inverse proposition does not hold, for example, for some real Daubechies wavelets, it is impossible to find the corresponding symmetric complexvalued ones [1], [18].

\section{B. Linear-Phase Property}

As mentioned earlier, both symmetry and linear-phase response are often desirable properties of digital filters. However, a given symmetric filter does not necessarily posses a linear-phase response, i.e., nonlinear-phase distortion may still exist in the output signals of the filter. Strictly speaking, a digital filter is called linear phase only if its phase-frequency response $\varphi(\omega)$ is a linear function of $\omega$, i.e., has the form $\alpha \cdot \omega$, where $\alpha$ is a constant. However, in engineering practice, a loose definition is often used [7], [22], i.e., a filter $h$ is said to have linear phase if

$$
H\left(e^{j \omega}\right)=c e^{j \alpha \omega} H_{R}(\omega)
$$

where

$c \quad$ complex constant;

$\alpha \quad$ real number;

$H_{R}(\omega)$ real function of $\omega$.

This definition shows that the phase response of the filter can be piecewise linear with possible phase jump $\pi$.

It is well known that if a real FIR filter $h$ is symmetric $(h[n]=h[N-1-n])$ or antisymmetric $(h[n]=-h[N-1-$ $n])$, then the filter has the linear-phase property [7], [21].

If an FIR filter $h$ has complex-valued coefficients, it can be shown (see Appendix A) that if $h$ is conjugate symmetric $(h[n]=\bar{h}[N-1-n])$ or conjugate antisymmetric $(h[n]=$ $-\bar{h}[N-1-n])$, then the filter has linear phase. However, a general nonreal complex-valued filter $h$ does not have linear phase if it is symmetric or antisymmetric.

Then, the natural question follows: Can we construct compactly supported linear phase dyadic complex-valued wavelet bases? In response, we present and prove the following theorem.

Theorem 2: The compactly supported dyadic orthogonal complex-valued wavelet bases cannot be linear phase.

Proof: Proving this theorem is equivalent to proving that a linear-phase complex-valued CQF PRFB that fulfills the conditions [3], [4] to construct a scaling function and wavelet does not exist. Assume there exists a linear-phase complexvalued CQF PRFB, i.e., its associated scaling function $h$ is linear phase; then, $h$ must be conjugate symmetric $(h[n]=$ $\bar{h}[N-1-n])$ or conjugate antisymmetric $(h[n]=-\bar{h}[N-$ $1-n])$.

Assume the length of the filter is $N$, i.e., $h[0] \neq 0$ and $h[N-1] \neq 0$, while $h[n]=0$ when $n<0$ or $n>N-1$. Since $N$ is even, we can assume $N=2 n_{0}$. Then, whether $h$ is conjugate symmetric or conjugate antisymmetric

$$
\begin{aligned}
\delta[k]= & \sum_{n} h[n] \bar{h}[n+2 k] \\
= & \sum_{m} h[2 m] \bar{h}[2 m+2 k] \\
& +\sum_{m} h[2 m-1] \bar{h}[2 m-1+2 k] \\
= & \sum_{m} h[2 m] \bar{h}[2 m+2 k] \\
& +\sum_{m} \bar{h}\left[2 n_{0}-2 m\right] h\left[2 n_{0}-2 m-2 k\right] \\
= & 2 \sum_{m} h[2 m] \bar{h}[2 m+2 k]
\end{aligned}
$$

holds for all $k$. Note that $\delta$ is a Kronecker $\delta$.

Let $\alpha[n]=h[2 m]$. Then, $\left|A\left(e^{j \omega}\right)\right|^{2}=1 / 2$, i.e., $\exists n^{\prime} \in \mathbf{Z}$ so that $\alpha[n]=c \delta\left[n-n^{\prime}\right]$. Thus, $\exists m^{\prime} \in \mathbf{Z}$ so that $h[2 m]=$ $c \delta\left[m-m^{\prime}\right]$, where $|c|=1 / \sqrt{2}$. Since $h[0] \neq 0$, we have $m^{\prime}=0$, i.e., $h[2 m]=c \delta[m]$; then, $h[N-1]=\bar{h}[0]=\bar{c}$. Hence, $h[2 m+1]=\bar{c} \delta\left[m-n_{0}\right]$. Subsequently, from the normalization condition $\left|\sum_{n} h[n]\right|=\sqrt{2}$, we get $c= \pm 1 / \sqrt{2}$. Therefore, it can be easily deduced that the scaling function can only be a real Haar function. This contradicts our original assumption, i.e., $h$ cannot be conjugate symmetric or conjugate antisymmetric.

Just as in the real-valued case, it is clear from Theorem 2 that the linear-phase complex CQF PRFB does not exist. However, it is possible to design the complex symmetric CQF with approximate linear phase by the following method.

First, design the real CQF PRFB, assuming the real scaling filter is $h^{\prime}$ with length $N$, and $P\left(e^{j \omega}\right)=\left|H^{\prime}\left(e^{j \omega}\right)\right|^{2}$. In the $z$ domain, $P(z)=H^{\prime}(z) H^{\prime}\left(z^{-1}\right)$.

Daubechies [22], [23] and Smith et al. [11] showed that $P(z)$ should have the form

$$
\begin{aligned}
P(z)= & C\left(1+z^{-1}\right)^{K} \prod_{m=1}^{M}\left(z^{-1}-r_{m}\right)\left(z^{-1}-r_{m}^{-1}\right) \\
& \cdot \prod_{j=1}^{J}\left(z^{-1}-z_{j}\right)\left(z^{-1}-z_{j}^{-1}\right)\left(z^{-1}-\bar{z}_{j}\right)\left(z^{-1}-\bar{z}_{j}^{-1}\right)
\end{aligned}
$$

where $r_{m}$ denotes the real zeros, and $z_{j}$ denotes the complex zeros. Its distribution of zeros is shown in Fig. 1. The zeros $z=-1$ represent the regularity of the scaling function. Note that when $K=N$, it becomes the Daubechies compactly supported scaling filter.

From Theorem 1, we can expect to construct the symmetric complex-valued CQF scaling filter $H(z)$ from $P(z)$. For CQF filter $H(z)$, as shown in [7] and [9], $P(z)=H(z) \tilde{H}(z)=$ $H(z) H_{*}\left(z^{-1}\right)$. This implies that if $z_{j}$ is a zero of $H(z)$, then $\bar{z}_{j}^{-1}$ will be a zero of $\tilde{H}(z)$.

For symmetric complex-valued CQF scaling filter $H(z)$, we should have $H(z)=z^{-(N-1)} H\left(z^{-1}\right)$, i.e., its zeros must be reciprocal pairs as $\left(z_{j}, z_{j}^{-1}\right)$.

Since $K$ is even, we can choose half the number of the zeros for $H(z)$ and the remaining half for $\tilde{H}(z)$. Note that 


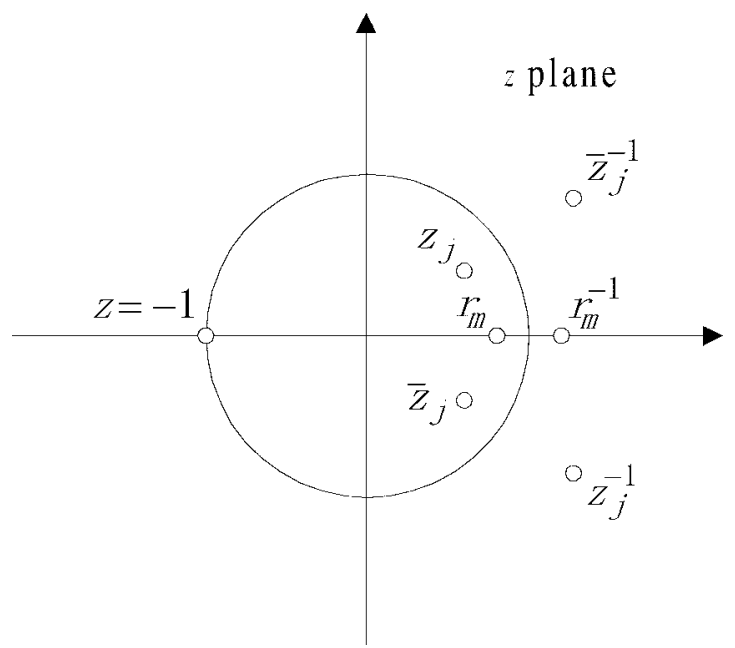

Fig. 1. Zeros distribution of $P(z)$.

the complex zeros of $P(z)$ are sets of four, $\left(z_{j}, z_{j}^{-1}, \bar{z}_{j}, \bar{z}_{j}^{-1}\right)$. The zeros of $H(z)$ can be selected as $z_{j}$ and $z_{j}^{-1}$ (or $\bar{z}_{j}$ and $\bar{z}_{j}^{-1}$ ), and the zeros of $\tilde{H}(z)$ can be selected as $\bar{z}_{j}$ and $\bar{z}_{j}^{-1}$ (or $z_{j}$ and $z_{j}^{-1}$ ). Only the real double roots (zeros) of $P(z)$ will appear as conjugate reciprocal sets of four. Similar to the complex zeros, one pair of reciprocal zeros $\left(r_{m}, r_{m}^{-1}\right)$ from the double roots can be selected for $H(z)$ and the other pair for $\tilde{H}(z)$. The single real roots $r_{m}$ and $r_{m}^{-1}$ of $P(z)$ cannot be decomposed as the reciprocal pair according to the above-mentioned requirements on $H(z)$ and $\tilde{H}(z)$.

However, sometimes $P(z)$ has no real zeros, and then, we can deduce the symmetric complex-valued CQF scaling filter $H(z)$ from $P(z)$ of the corresponding real-valued CQF scaling filter $H^{\prime}(z)$.

As mentioned in [1] and [18], when $K=N$ for Daubechies CQF filters, $2 M+4 J=N-2$. If $N$ is an integral multiple of 4 (notice that $N$ must be even), then there must exist the reciprocal pair of the single roots of $P(z)$. Therefore, the corresponding complex CQF filter cannot be constructed from the real CQF filter. On the other hand, when $N$ is not a multiple of 4 , whether the corresponding complex CQF filters can be deduced from all Daubechies real CQF filters is still an open question.

Without loss of generality, assume that the complex zeros $z_{j}, j=1, \ldots, J$ of $P(z)$ are within the unit circle and the upper half of the $z$ plane, with the phase angle $0<\operatorname{ang}\left(z_{1}\right)<$ $\operatorname{ang}\left(z_{2}\right)<\cdots<\operatorname{ang}\left(z_{j}\right)<\pi$. When decomposing the zeros of $P(z)$, if all $z_{j}, j=1,2, \ldots, J$ are selected for $H(z)$, then the normal symmetric complex CQF filter is obtained. Note that this will be a special case of Daubechies CQF filters. Lawton obtained symmetric complex Daubechies CQF filters using this method [1]. However, if odd indexed zeros of $z_{j}$, $j=1, \ldots, J$ are selected for $H(z)$ and even indexed zeros are selected for $\tilde{H}(z)$, then approximate linear-phase symmetric complex CQF filter are obtained because a linear-phase filter has conjugate reciprocal zero pairs $\left(z, \bar{z}^{-1}\right)$. This scheme is similar to the one used by Smith et al. [11] and Daubechies [22] for the real-valued case.

The above-mentioned method can be used to design not only Daubechies minimum compactly supported symmetric com-
TABLE I

First Column Is the Normal Coefficients of Complex Symmetric (NSC) Daubechies CQF Scaling Filter and the Second Column Is the Coefficients of Complex ApProximately Linear-Phase Symmetric (ALPSC) Daubechies CQF Scaling Filter, Where $h[n]=h[N-1-n]$

\begin{tabular}{|c|c|c|c|}
\hline $\bar{N}$ & $n$ & $h[n](\mathrm{NSC})$ & $h[n]$ (ALPSC) \\
\hline \multirow[t]{3}{*}{6} & 0 & $-0.0662912607+0.0855816496 j$ & \\
\hline & 1 & $0.1104854346+0.0855816496 j$ & \\
\hline & 2 & $0.6629126074-0.1711632992 j$ & \\
\hline \multirow[t]{5}{*}{10} & 0 & $-0.0131549131-0.0190001547 j$ & $0.0104924505+0.0205904371 \mathrm{j}$ \\
\hline & 1 & $-0.0407762717+0.0282317864 j$ & $-0.0171289081+0.0087285287 j$ \\
\hline & 2 & $0.0139497502+0.2176964423 j$ & $-0.0806397041-0.1179474735 j$ \\
\hline & 3 & $0.2459691628+0.1232325600 \mathrm{j}$ & $0.1513797084-0.0942236567 j$ \\
\hline & 4 & $0.5011190530-0.3501606340 \mathrm{j}$ & $0.6430032345+0.1828521645 j$ \\
\hline \multirow[t]{7}{*}{14} & 0 & $0.0049120149-0.0018464710 \mathrm{j}$ & $-0.0019561120+0.0048693913 j$ \\
\hline & 1 & $-0.0054111299-0.0143947836 j$ & $0.0032381460+0.0013008148 j$ \\
\hline & 2 & $-0.0701089996-0.0079040001 j$ & $0.0176519705-0.0376015185 j$ \\
\hline & 3 & $-0.0564377788+0.1169376946 j$ & $-0.0307464201-0.0140449767 j$ \\
\hline & 4 & $0.1872348173+0.2596312614 j$ & $-0.0864785180+0.1308596826 j$ \\
\hline & 5 & $0.3676385056+0.0475928095 j$ & $0.1715121845+0.0944523287 j$ \\
\hline & 6 & $0.2792793518-0.4000165107 j$ & $0.6338855303-0.1798357222 j$ \\
\hline \multirow[t]{9}{*}{18} & 0 & $0.0001673289+0.0012125454 j$ & $0.0003942226+0.0011588157 j$ \\
\hline & 1 & $0.0044674339-0.0006164972 j$ & $-0.0006617029+0.0002$ \\
\hline & 2 & $0.0062198948-0.0211481356 j$ & $-0.0042292751-0.0112272165 j$ \\
\hline & 3 & $-0.0440552941-0.0275890806 j$ & $0.0073680018-0.0027405777 j$ \\
\hline & 4 & $-0.1194569938+0.0742727829 j$ & $0.0225529709+0.0499330794 j$ \\
\hline & 5 & $0.0110213530+0.2211650683 j$ & $-0.0408982409+0.0174301294 j$ \\
\hline & 6 & $0.3474269887+0.1685984877 j$ & $-0.0897522960-0.1361570402 j$ \\
\hline & 7 & $0.3968721371-0.0985470780 \mathrm{j}$ & $0.1833586940-0.0928762244 j$ \\
\hline & 8 & $0.1044439327-0.3173480928 j$ & $0.6289744068+0.1742539267 j$ \\
\hline \multirow[t]{11}{*}{22} & 0 & $-0.0002890832-0.0000211708 j$ & $-0.0000831451+0.0002776764 j$ \\
\hline & 1 & $-0.0000935982+0.0012780664 j$ & $0.0001411949+0.0000422782 j$ \\
\hline & 2 & $0.0059961342+0.0029648612 j$ & $0.0010437781-0.0032380120 \mathrm{j}$ \\
\hline & 3 & $0.0122232015-0.0144283733 j$ & $-0.0018308635-0.0005859558 j$ \\
\hline & 4 & $-0.0243700791-0.0503067404 j$ & $-0.0063536692+0.0175220001 j$ \\
\hline & 5 & $-0.1092940542+0.00446$ & $0.0114832592+0.0040506389 j$ \\
\hline & 6 & $-0.0918847036+0.1999654035 j$ & $0.0261082799-0.058778$ \\
\hline & 7 & $0.1540094645+0.2603015239 j$ & $-0.0486011747-0.01972$ \\
\hline & 8 & $0.4014277015-0.0013800055 j$ & $-0.0919600615+0.1380002004 j$ \\
\hline & 9 & $0.3153022916-0.2232934469 j$ & $0.1911016248+0.0908806184 j$ \\
\hline & 10 & $0.0440795062-0.1795460286 j$ & $0.6260575582-0.1684436948 j$ \\
\hline \multirow[t]{13}{*}{26} & 0 & $0.0000188880-0.0000666842 j$ & $0.0000180658+0.0000669117 j$ \\
\hline & 1 & $-0.0003432243-0.0000972169 j$ & $-0.0000309778+0.0000083639 j$ \\
\hline & 2 & $-0.0011728503+0.0016006744 j$ & $-0.0002607987-0.0009126780 j$ \\
\hline & 3 & $0.0042233619+0.0048993464 j$ & $0.0004606252-0.0001310241 j$ \\
\hline & 4 & $0.0196555369-0.0066509422 j$ & $0.0018115864+0.0058273044 j$ \\
\hline & 5 & $0.0016797833-0.0476733253 j$ & $-0.0032888907+0.0010064679 j$ \\
\hline & 6 & $-0.0953962269-0.0487974312 j$ & $-0.0082348462-0.0232218672 j$ \\
\hline & 7 & $-0.1479282408+0.1025689720 j$ & $0.0152897663-0.0051889009 j$ \\
\hline & 8 & $0.0310224644+0.2893865149 j$ & $0.0288266812+0.0651864904 j$ \\
\hline & 9 & $0.2980363862+0.1896532730 j$ & $-0.0546003089+0.0213655054 j$ \\
\hline & 10 & $0.3322438272-0.1486773856 j$ & $-0.0936227228-0.1381234283 j$ \\
\hline & 11 & $0.1796833483-0.2555307427 j$ & $0.1965231655-0.0888539941 \mathrm{j}$ \\
\hline & 12 & $0.0853837273-0.0806150524 j$ & $0.6242154359+0.1629708492 j$ \\
\hline
\end{tabular}

plex CQF filters but any other approximate linear-phase symmetric complex CQF filters as well. Next, we use Daubechies class of CQF filters as an example to analyze the phase of different types of CQF filters.

\section{Numerical Analysis of Phase Property of Daubechies Wavelets}

Some design results are presented in Table I for complex Daubechies wavelets. In Table I, the coefficients of the normal symmetric complex (NSC) Daubechies CQF scaling filters are shown in the left column, and the approximately linear phase complex (ALPSC) ones are shown in the right column. When $N=6$, there is only one complex conjugate reciprocal pair 


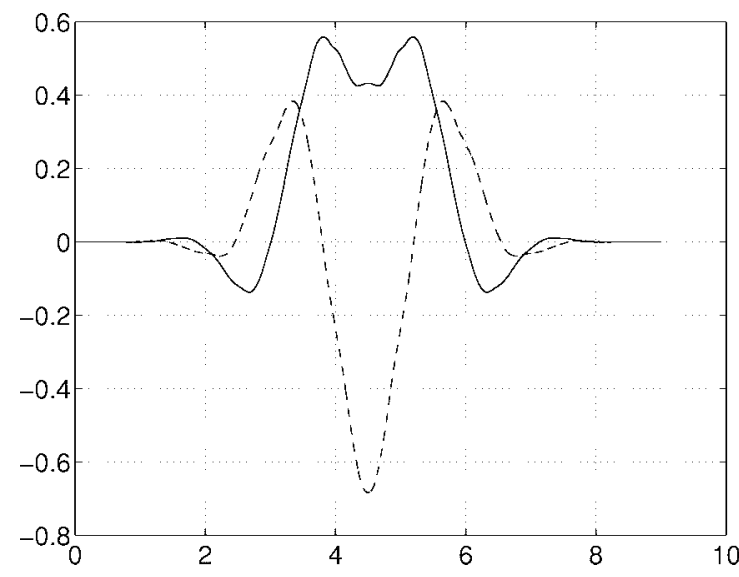

(a)

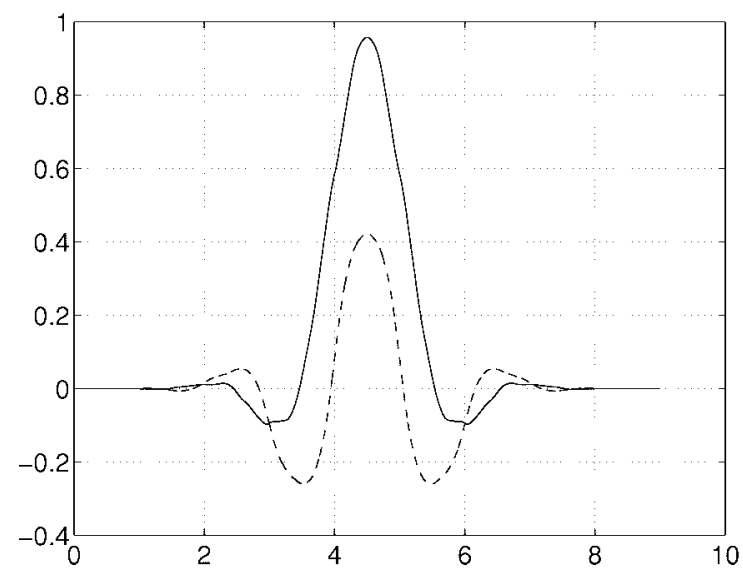

(c)

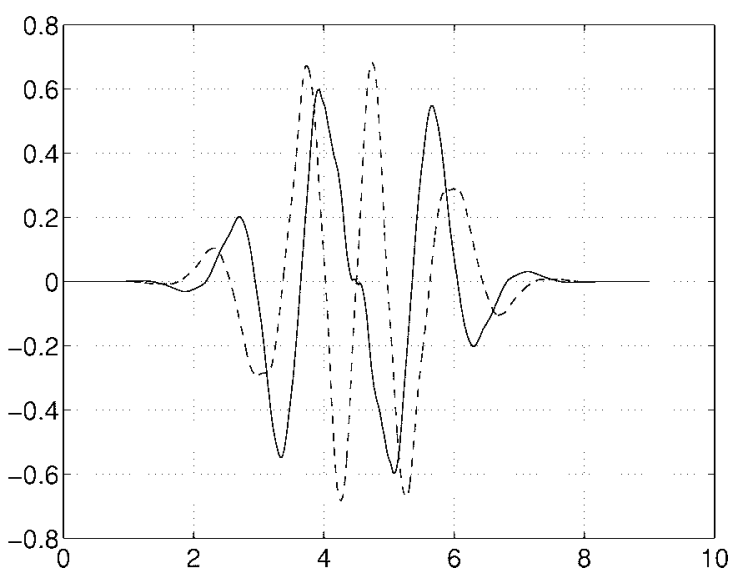

(b)

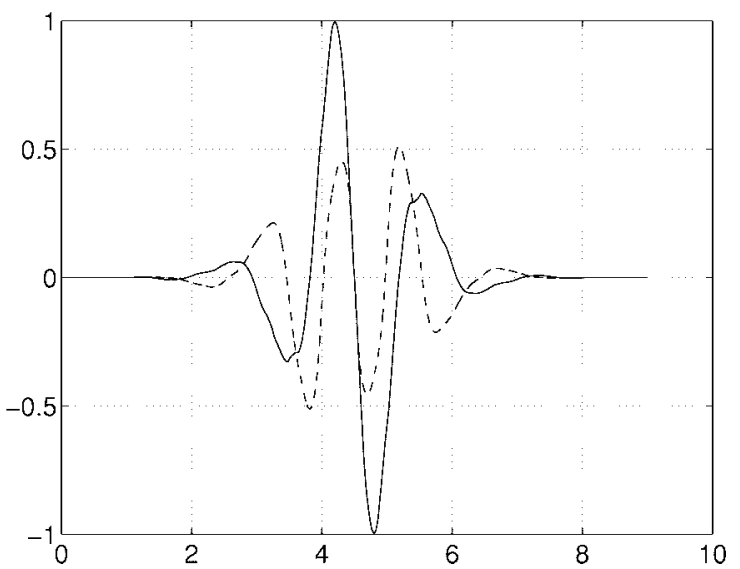

(d)

Fig. 2. Symmetric complex Daubechies scaling functions and wavelets for $N=10$. The solid line shows real part, and the dashed line shows imaginary part. (a) NSC Daubechies scaling function. (b) NSC Daubechies wavelet. (c) ALPSC Daubechies scaling function. (d) ALPSC Daubechies wavelet.

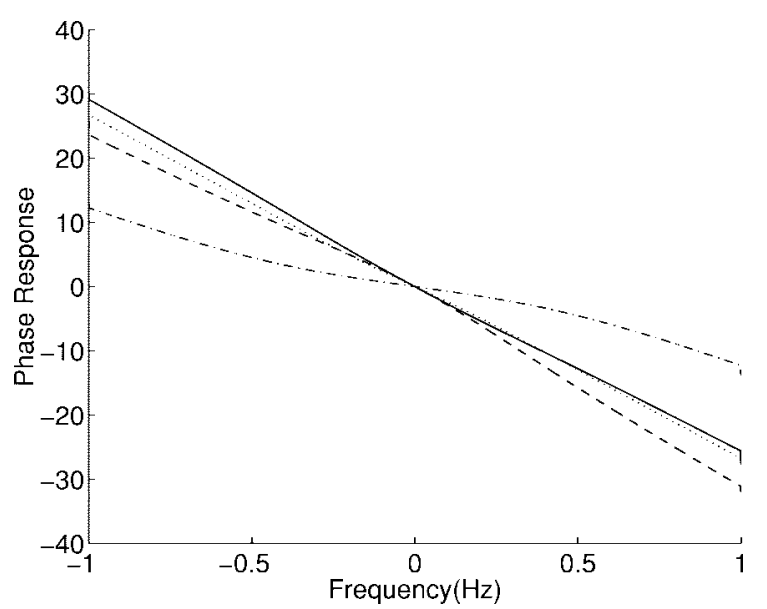

Fig. 3. Phase-frequency response of Daubechies scaling functions for $N=10$. The solid line shows the phase response of ALPSC; the dashed line shows NSC; the dotted line shows ALPR, and the dash-dotted line shows NR.

for $P(z)$; therefore, there is only one decomposition method to derive symmetric $H(z)$.

In Fig. 2, the NSC and ALPSC scaling functions and wavelets when $N=10$ are shown. The solid line represents the real part, whereas the dashed line represents the imaginary part.
TABLE II

Linear Correlation Coefficients of the Phase Responses of Different Daubechies Scaling Functions

\begin{tabular}{c|c|c|c|c}
\hline$N$ & ALPSC & NSC & NR & ALPR \\
\hline 10 & 0.99579 & 0.98041 & 0.98726 & 0.99972 \\
\hline 14 & 0.99772 & 0.97459 & 0.98377 & 0.99981 \\
\hline 18 & 0.99863 & 0.97082 & 0.98258 & 0.99982 \\
\hline 22 & 0.99902 & 0.96777 & 0.98148 & 0.99994 \\
\hline 26 & 0.99931 & 0.96591 & 0.98119 & 0.99988 \\
\hline 30 & 0.99945 & 0.96378 & 0.97759 & 0.99998 \\
\hline 34 & 0.99956 & 0.96222 & 0.97995 & 0.99995 \\
\hline 38 & 0.99963 & 0.96339 & 0.98001 & 0.99974 \\
\hline
\end{tabular}

The phase responses of different Daubechies scaling functions with $N=10$ are shown in Fig. 3. The ALPSC is shown as the solid line; the NSC is shown as dashed line; In addition, the phase response of the corresponding normal real (NR) Daubechies scaling functions and the phase response of the approximately linear-phase real (ALPR) Daubechies scaling functions [22] are shown as dash-dotted and dotted lines, respectively. In this figure, the phase-frequency response of the scaling functions are calculated for 1024 samples and the range of $[-2 \pi, 2 \pi]$. Since the scaling functions are lowpass, the range of $[-2 \pi, 2 \pi]$ is sufficient. Note that these phase responses are compensated by the phase jump of $\pi$. 


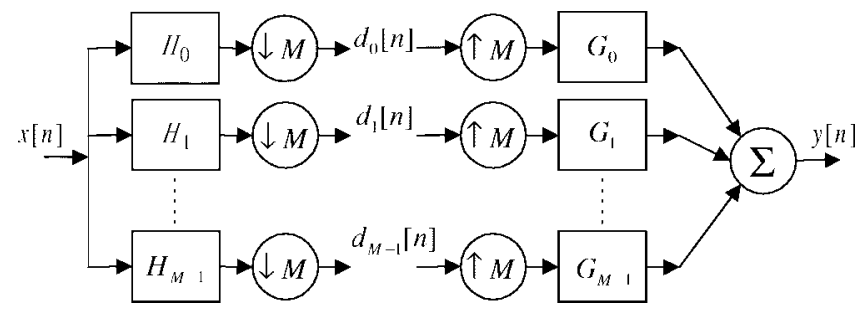

Fig. 4. Structure of M-band FIR filter bank.

The linear correlation coefficients are also calculated, as shown in Table II. From Table II and Fig. 3, we see that the ALPR Daubechies scaling function possesses the best approximation to linear phase, and the NSC one has the worst linear phase: even worse than the normal real Daubechies wavelet. The ALPSC is a good choice if both symmetry and approximately linear-phase properties are desired.

In a recent paper [24], Lina's independent work used another method to obtain symmetric Daubechies wavelets (SDW's) $J$, which give the same results as our ALPSC results using Daubechies wavelets. However, our ALPSC wavelets are more general; they can be any type of compactly supported orthogonal wavelets, including the Daubechies class.

\section{The Design of General OrthogOnal COMPACTLY SUPPORTED COMPLEX WAVELETS}

As seen in Section II, symmetric complex CQF filters can only have the same amplitude spectra as real CQF filters. This implies that their amplitude spectra must be an even function. However, some applications require asymmetric amplitude spectra (with respect to the zero frequency) when processing the complex I/Q orthogonal signal. For example, the general Morlet wavelet has asymmetric amplitude spectrum. In this sense, the frequency response of the complex filter is more flexible than the real filter. Therefore, for some applications, design of the general orthogonal compactly supported complex wavelet may be necessary.

Designing orthogonal compactly supported complex wavelets is equivalent to designing the associated complex CQF PRFB's. In the following, we will develop a parameterization method of general complex CQF PRFB's that will be used to design complex CQF PRFB's.

\section{A. The Parameterization of General Complex CQF PRFB's}

The structure of $M$-band FIR FB's is depicted in Fig. 4.

Analogous to the two-band case, for $M$-band PRFB's, if filter $h_{0}$ satisfies

$$
\begin{aligned}
\sum_{k} h_{0}[k] \bar{h}_{0}[k+M l] & =\delta[l] \\
\left|\sum_{k} h_{0}[k]\right| & =\sqrt{M}
\end{aligned}
$$

then $h_{0}$ can be the $M$-band scaling filter associated with an $M$-band multiresolution analysis and, therefore, can be used to construct $M$-band wavelet bases [7], [25], [26].

To develop the parameterization method of general $M$-band complex CQF PRFB's. We will require the following Lemma.
Lemma 1: Assume $a_{1}, a_{2}, \ldots, a_{N} \in \mathbf{C}$; then

$$
\frac{\left|\sum_{k=1}^{N} a_{k}\right|}{N} \leq \sqrt{\frac{\sum_{k=1}^{N}\left|a_{k}\right|^{2}}{N}}
$$

holds, where the equality holds if and only if $a_{1}=a_{2}=\cdots=$ $a_{N}$. (See Appendix B for proof).

In the following theorem, we give the Householder decomposition of the $M$-band scaling filter. Note that it is similar to the real $M$-band scaling filter.

Theorem 3: Assume that $h_{0}$ is a scaling filter with length $N$ associated with $M$-band complex orthogonal wavelet bases. Its $z$ transform can be written in polyphase form as

$$
H_{0}(z)=\sum_{k=0}^{M-1} z^{-k} H_{0, k}\left(z^{M}\right) .
$$

Define the polyphase vector as

$$
\mathbf{h}_{0}(z)=\left[H_{0,0}, H_{0,1}, \ldots, H_{0, M-1}\right]^{T} .
$$

Define the $M \times 1$ complex constant vector

$$
\mathbf{v}_{0}=e^{j \varphi_{0}} \cdot\left[\frac{1}{\sqrt{M}}, \frac{1}{\sqrt{M}}, \ldots, \frac{1}{\sqrt{M}}\right] .
$$

Then, the Householder decomposition form

$$
\mathbf{h}_{0}(z)=\mathbf{V}_{N}(z) \mathbf{V}_{N-1}(z) \cdots \mathbf{V}_{1}(z) \mathbf{v}_{0}(z)
$$

holds for $h_{0}$, and

$$
\left|\sum_{k} h_{0}[M k+n]\right|=\frac{1}{\sqrt{M}}, \quad \forall n \in \mathbf{Z}
$$

where

$$
\mathbf{V}_{n}(z)=\mathbf{I}-\mathbf{v}_{n} \mathbf{v}_{n}^{H}+z^{-1} \mathbf{v}_{n} \mathbf{v}_{n}^{H}
$$

and $\mathbf{v}_{n}$ is an $M \times 1$ complex vector that satisfies $\left\|\mathbf{v}_{n}\right\|=1$.

Proof: Assume that $a(l)=\sum_{k} h_{0}[k] \bar{h}_{0}[k+M l]=\delta[l]$; then, its $z$ transform becomes

$$
\begin{aligned}
1=A(z) & =[\downarrow M] H_{0}(z) \tilde{H}_{0}(z) \\
& =[\downarrow M] \sum_{k, j} z^{-k} H_{0, k}\left(z^{M}\right) z^{j} \tilde{H}_{0, j}\left(z^{M}\right) \\
& =\sum_{k} H_{0, k}(z) \cdot \tilde{H}_{0, k}(z)
\end{aligned}
$$

i.e., $\mathbf{h}_{0}(z)$ is a unitary polynomial vector. Hence [7], we have

$$
\mathbf{h}_{0}(z)=\mathbf{V}_{N}(z) \mathbf{V}_{N}(z) \cdots \mathbf{V}_{1}(z) \mathbf{u}_{0}(z)
$$


where $\mathbf{V}_{n}(z)$ is defined as (17) $\mathbf{u}_{0} \in \mathbf{C}^{M \times 1}$ and $\left\|\mathbf{u}_{0}\right\|=1$. Using (17), we get

$$
\begin{aligned}
\sqrt{M} & =\left|\sum_{k} h_{0}[k]\right|=\left|H_{0}(z)\right|_{z=1} \mid \\
& =\left|\sum_{k} z^{-k} H_{0, k}\left(z^{M}\right)\right|_{z=1}|=| \sum_{k} H_{0, k}(z) \mid .
\end{aligned}
$$

Since for $\mathbf{V}_{n}(z)$ we have

$$
\left.\mathbf{V}_{n}(z)\right|_{z=1}=\left.\left[\mathbf{I}-\mathbf{v}_{n} \mathbf{v}_{n}^{H}+z^{-1} \mathbf{v}_{n} \mathbf{v}_{n}^{H}\right]\right|_{z=1}=\mathbf{I}
$$

then $\mathbf{h}_{0}(1)=u_{0}$. From (20) and (13), we get

$$
\left|\sum_{k} u_{0, k}\right|=\left|\sum_{k} H_{0, k}(z)\right| z=1 \mid=\sqrt{M}
$$

Since $\left\|\mathbf{u}_{0}\right\|=\sqrt{\sum_{k}\left|u_{0, k}\right|^{2}}=1$, from Lemma 1, the solution of $\mathbf{u}_{0}$ must be equal to $\mathbf{v}_{0}$ in (14). Since $\mathbf{h}_{0}(1)=\mathbf{u}_{0}$, using (13) and (22), we know (16) holds.

It is also easy to prove that the $h_{0}$ as in (14) and (15) satisfies the conditions of (9) and (10).

By using this theorem, we can get the parameterization of the scaling filter associated with general $M$-band complex orthogonal wavelet bases. We take the elements of vector $\mathbf{v}_{n}$, $n=1,2, \ldots, N / M$ in (17) as (23), shown at the bottom of the page, i.e., $\mathbf{v}_{n}$ can be determined by $2(M-1)$ angle parameters $\theta_{n, j}, \varphi_{n, j}, j=0,1, \ldots, M-2$.

After we get the scaling filter, we also can construct the other complex wavelet filters by the method in [26].

Obviously, dyadic complex orthogonal wavelet bases are a special cases of the $M$-band complex orthogonal wavelet bases, i.e., we can use the method in the above section by taking $M=2$. Then, the wavelet filter $g$ can be simply determined by $g[k]=(-1)^{k} \bar{h}[N-1-k]$ with filter length $N$. Then, (23) becomes

$$
\mathbf{v}_{n}=\left[\cos \left(\theta_{n}\right) \exp \left(j \varphi_{n}\right), \sin \left(\theta_{n}\right)\right]^{T}, \quad n=1,2, \ldots, N / 2 .
$$

\section{B. Some Examples}

In Table III, we give the coefficients of dyadic $(M=2)$ complex scaling filter with $N=4$, which are calculated by (14), (15), and (24). As an example, we fix $\theta_{1}=5 \pi / 6$, $\varphi_{0}=0$ and vary $\varphi_{1}$. Note that $\varphi_{1}=0$ corresponds to a real Daubechies filter. The amplitude spectra $\left|H\left(e^{j \omega}\right)\right|$ of the scaling filters are shown in Fig. 5(a)-(e). The filters with various asymmetric amplitude spectrums can be designed by
TABLE III

Two-Band Complex CQF Scaling Filter Coefficients Determined by Angle Parameter $\varphi_{1}$ When $N=4$

\begin{tabular}{c|r|r}
\hline$\varphi_{1}$ & $n$ & \multicolumn{1}{|c}{$h[n]$} \\
\hline 0 & 0 & 0.4829629131 \\
& 1 & 0.8365163037 \\
& 2 & 0.2241438680 \\
& 3 & -0.1294095226 \\
\hline$-\frac{\pi}{8}$ & 0 & $0.4596558751-0.1171723928 \mathrm{j}$ \\
& 1 & $0.8132092657+0.1171723928 \mathrm{j}$ \\
& 2 & $0.2474509061+0.1171723928 \mathrm{j}$ \\
& 3 & $-0.1061024845-0.1171723928 \mathrm{j}$ \\
\hline$-\frac{\pi}{4}$ & 0 & $0.3932830462-0.2165063509 \mathrm{j}$ \\
& 1 & $0.7468364368+0.2165063509 \mathrm{j}$ \\
& 2 & $0.3138237349+0.2165063509 \mathrm{j}$ \\
& 3 & $-0.0397296556-0.2165063509 \mathrm{j}$ \\
\hline$-\frac{3 \pi}{8}$ & 0 & $0.2939490881-0.2828791798 \mathrm{j}$ \\
& 1 & $0.6475024787+0.2828791798 \mathrm{j}$ \\
& 2 & $0.4131576931+0.2828791798 \mathrm{j}$ \\
& 3 & $0.0596043025-0.2828791798 \mathrm{j}$ \\
\hline$-\frac{\pi}{2}$ & 0 & $0.1767766953-0.3061862178 \mathrm{j}$ \\
& 1 & $0.5303300859+0.3061862178 \mathrm{j}$ \\
& 2 & $0.5303300859+0.3061862178 \mathrm{j}$ \\
& 3 & $0.1767766953-0.3061862178 \mathrm{j}$ \\
\hline
\end{tabular}

changing angle parameters. Similarly, the waveshape of the filter amplitude spectrums can also be adapted by changing the angle parameters $\theta_{n}$.

For general $(M \neq 2)$ compactly supported $M$-band complex orthogonal wavelet bases and their associated complex orthogonal FIR CQF PRFB's [27], we can also change the waveshape of the frequency response of filters flexibly by using the parameterization method expressed by (14), (15), and (23). After the scaling filter $h_{0}$ is designed, then the method in [7], [25], and [26] can be used to construct other wavelet filters in $M$-band complex orthogonal filter banks.

\section{CONCLUSION}

In this paper, first we investigated the symmetry and linearphase property of compactly supported complex orthogonal wavelet bases and their associated complex orthogonal FIR $\mathrm{CQF} F B$ and proved that it is impossible to construct linearphase compactly supported complex orthogonal wavelet bases. However, both symmetry and linear phase are important and useful properties in practice. We proposed a scheme to construct the symmetric compactly supported complex orthogonal wavelet base with approximately linear phase. The phase response of different kinds of Daubechies scaling functions are analyzed numerically. It is shown that the phase responses of normal symmetric complex Daubechies scaling functions, which are often treated as linear phase [1], [2], have the smallest linear correlation coefficients.

Both the real orthogonal wavelet and the symmetric complex orthogonal wavelet can only have symmetric amplitude

$$
v_{n, j}= \begin{cases}{\left[\prod_{k=0}^{j-1} \sin \left(\theta_{n, k}\right)\right] \cos \left(\theta_{n, j}\right) \exp \left(j \varphi_{n, j}\right),} & j=0,1, \ldots, M-2 \\ \prod_{k=0}^{M=2} \sin \left(\theta_{n, k}\right), & j=M-1\end{cases}
$$




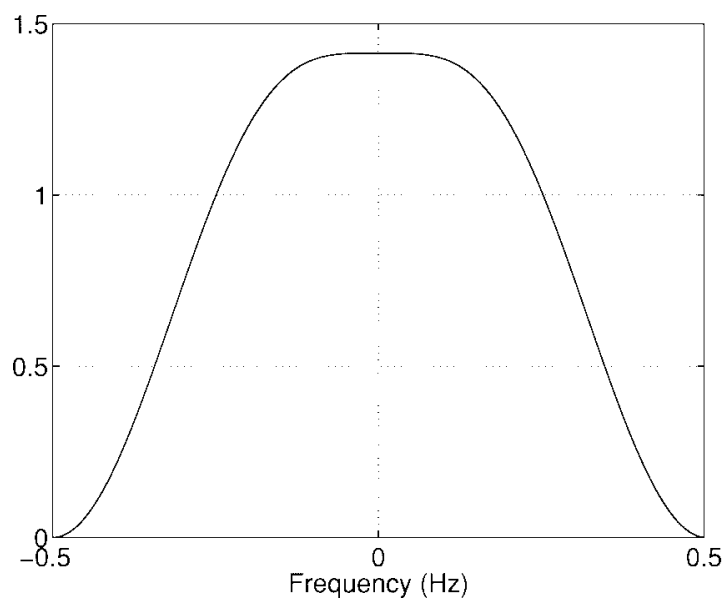

(a)

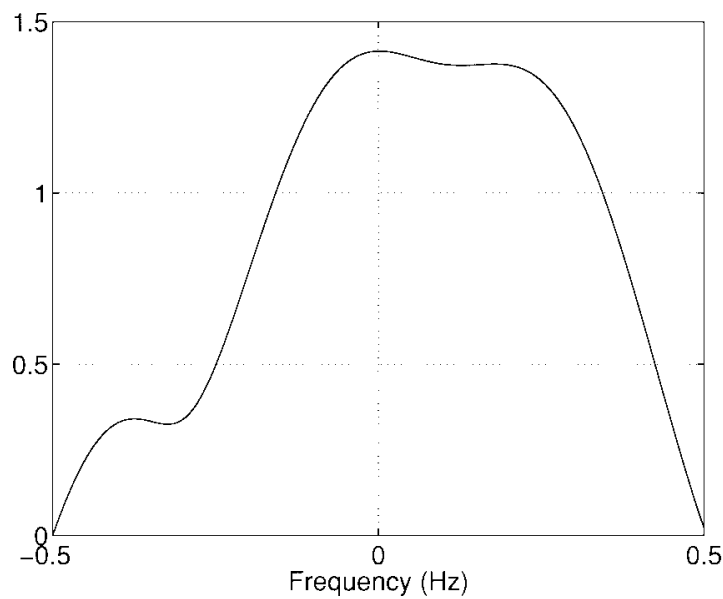

(c)

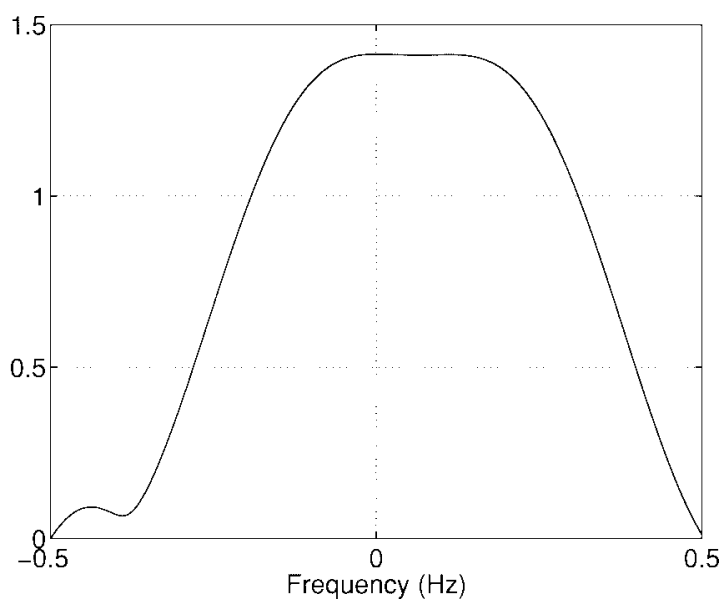

(b)

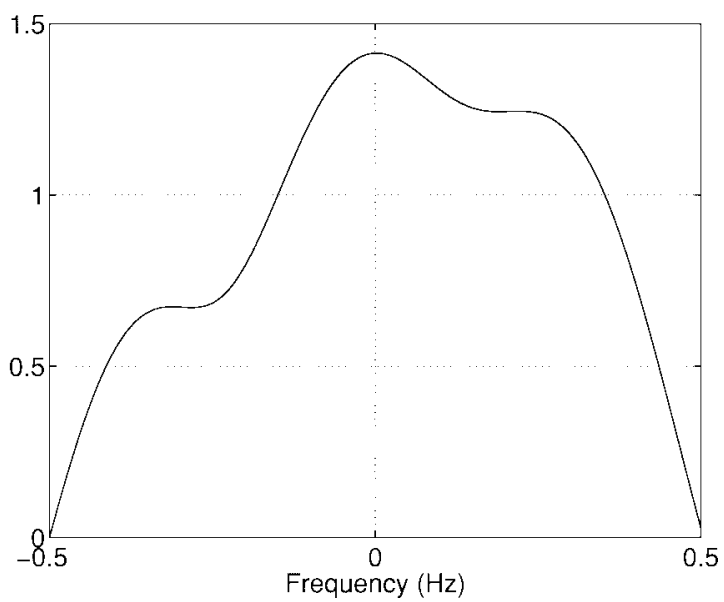

(d)

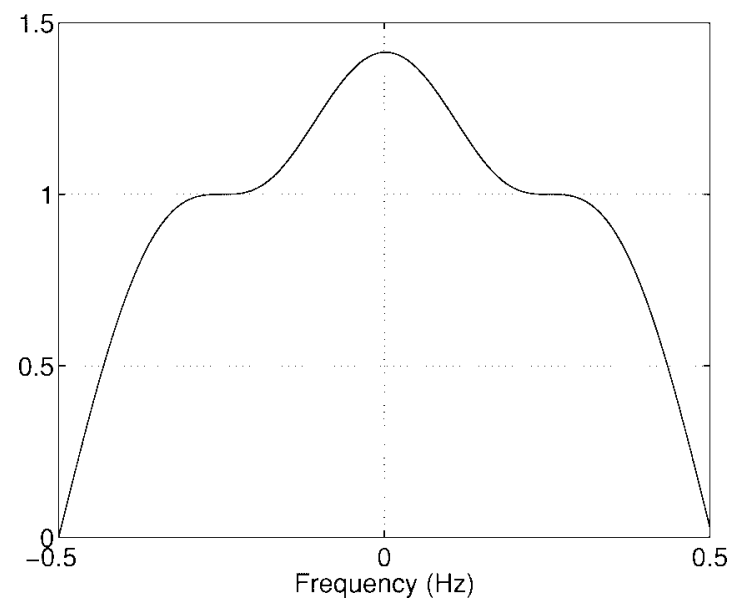

(e)

Fig. 5. Filter amplitude-frequency response $\left|H\left(e^{j \omega}\right)\right|$ of the complex CQF scaling filters with different angle parameter. (a) $\varphi_{1}=0$. (b) $\varphi_{1}=-\frac{\pi}{8}$. (c) $\varphi_{1}=-\frac{\pi}{4}$. (d) $\varphi_{1}=-\frac{3 \pi}{8}$. (e) $\varphi_{1}=-\frac{\pi}{2}$.

spectra. However, in practice, for the general complex signals, asymmetric amplitude spectra are often needed. To this end, we developed a method to design general complex orthogonal filter banks and their associated wavelet bases by a parameterization scheme of complex orthogonal filter banks. Note that this is similar to their real-valued cousin. Some design examples were given. The design results show that the waveshape of complex wavelet bases and their associated filters can be easily adjusted by changing the angle parameters.

In analogy with real filter banks [7], [9], the parameterization of complex filter banks can also provide an effective structure and algorithm for implementation. Furthermore, it 
can be used as an effective method for selecting optimal wavelet bases and filter banks. The parameterization of complex filter banks changes the constrained optimization problem to an unconstrained problem, which can be easily solved by numerical algorithms. An appropriate criterion can be chosen and a similar optimization method used in choosing real wavelet bases [28]-[30] and can be used to choose optimal complex orthogonal wavelet bases and filter banks. Similarly, the method can also be used to select optimal complex wavelet packet bases.

\section{APPENDIX A}

Assume filter $h$ has even length $N$, and $h$ is conjugate symmetric $(h[n]=\bar{h}[N-1-n])$; then

$$
\begin{aligned}
H(z) & =\sum_{n=0}^{N-1} h[n] z^{-n} \\
& =\sum_{n=0}^{\frac{N}{2}-1} h[n] z^{-n}+\sum_{n=\frac{N}{2}}^{N-1} h[n] z^{-n} \\
& =\sum_{n=0}^{\frac{N}{2}-1} h[n] z^{-n}+\sum_{n=0}^{\frac{N}{2}-1} h[N-1-n] z^{N-1-n} \\
& =\sum_{n=0}^{\frac{N}{2}-1}\left[h[n] z^{-n}+\bar{h}[n] z^{N-1-n}\right] .
\end{aligned}
$$

The frequency response is then

$$
\begin{aligned}
H\left(e^{j \omega}\right)= & \sum_{n=0}^{\frac{N}{2}-1}\left[h[n] e^{-j \omega n}+\bar{h}[n] e^{-j \omega(N-1-n)}\right] \\
= & e^{-j \omega\left(\frac{N}{2}-1\right)} \sum_{n=0}^{\frac{N}{2}-1}\left[h[n] e^{j \omega\left(\frac{N}{2}-1-n\right)}\right. \\
& \left.+\bar{h}[n] e^{-j \omega\left(\frac{N}{2}-1-n\right)}\right] \\
= & e^{-j \omega\left(\frac{N}{2}-1\right)} \sum_{n=0}^{\frac{N}{2}-1} 2 \operatorname{Re}\left[h[n] e^{j \omega\left(\frac{N}{2}-1-n\right)}\right] \\
= & e^{-j \alpha \omega} H_{R}(\omega)
\end{aligned}
$$

where

$$
\begin{aligned}
\alpha & =\frac{N}{2}-1 \\
H_{R}(\omega) & =\sum_{n=0}^{\frac{N}{2}-1} 2 \operatorname{Re}\left[h[n] e^{j \omega\left(\frac{N}{2}-1-n\right)}\right] .
\end{aligned}
$$

This is the same form as in (6), i.e., filter $h$ is linear phase.

However, if $h$ is only symmetric $(h[n]=h[N-1-n])$, then (26) becomes

$H\left(e^{j \omega}\right)=e^{-j \omega\left(\frac{N}{2}-1\right)} \sum_{n=0}^{\frac{N}{2}-1}\left[2 h[n] \cos \left(\frac{N}{2}-1-n\right) \omega\right]$.

Obviously, when $h$ is a general complex series, the above cannot satisfy the linear-phase form of (6).
Similar results can be shown when $N$ is odd or when $h$ is conjugate symmetric $(h[n]=-\bar{h}[N-1-n])$. In fact, when an FIR filter with complex coefficients is linear phase, its zeros should appear as conjugate reciprocal pairs $\left(z_{0}, \bar{z}_{0}^{-1}\right)$.

\section{APPENDIX B \\ PROOF OF LEMMA 1}

Proof: The inequality in Lemma 1 is equivalent to

$$
\left|\sum_{k=1}^{N} a_{k}\right|^{2} \leq N \sum_{k=1}^{N}\left|a_{k}\right|^{2}
$$

Since $\left|a_{1}-a_{2}\right|^{2}=\left|a_{1}\right|^{2}+\left|a_{2}\right|^{2}-2 \operatorname{Re}\left\{a_{1} \bar{a}_{2}\right\} \geq 0$, i.e., $2 \operatorname{Re}\left\{a_{1} \bar{a}_{2}\right\} \leq\left|a_{1}\right|^{2}+\left|a_{2}\right|^{2}$ and if and only if $a_{1}=a_{2}$ the equality holds. Thus

$$
\begin{aligned}
\left|\sum_{k=1}^{N} a_{k}\right|^{2} & =\sum_{k=1}^{N}\left|a_{k}\right|^{2}+2 \sum_{i<j \leq N} \operatorname{Re}\left\{a_{i} \bar{a}_{j}\right\} \\
& \leq \sum_{k=1}^{N}\left|a_{k}\right|^{2}+\sum_{i<j \leq N}\left(\left|a_{i}\right|^{2}+\left|a_{j}\right|^{2}\right) \\
& =N \sum_{k=1}^{N}\left|a_{k}\right|^{2}
\end{aligned}
$$

where equality holds if and only if $a_{1}=a_{2}=\cdots=a_{N}$.

\section{REFERENCES}

[1] W. Lawton, "Applications of complex valued wavelet transforms to subband decomposition," IEEE Trans. Signal Processing, vol. 41, pp. 3566-3568, Dec. 1993.

[2] B. Belzer, J.-M. Lina, and J. Villasenor, "Complex, linear-phase filters for efficient image coding," IEEE Trans. Signal Processing, vol. 43, pp. 2425-2427, Oct. 1995.

[3] S. Mallat, "A theory for multiresolution signal decomposition: The wavelet representation," IEEE Trans. Pattern Anal. Machine Intell., vol. 11, pp. 674-693, July 1989.

[4] _ "Multiresolution approximations and wavelet orthonormal bases of $L 2(R)$," Trans. Amer. Math. Soc., vol. 315, no. 1, pp. 69-87, Sept. 1989.

[5] A. N. Akansu and R. A. Haddad, Multiresolution Signal Decomposition. New york: Academic, 1992

[6] M. Vetterli and J. Kovacevic, Wavelets and Subband Coding. Englewood Cliffs, NJ: Prentice-Hall, 1995.

[7] P. P. Vaidyanathan, Multirate Systems and Filter Banks. Englewood Cliffs, NJ: Prentice-Hall, 1993.

[8] , "Theory and design of $M$-channel maximally decimated quadrature mirror filters with arbitrary $M$, having perfect reconstruction properties," IEEE Trans. Acoust., Speech, Signal Processing, vol. ASSP35, pp. 476-492, Apr. 1987.

[9] _ "Multirate digital filters, filter banks, polyphase networks, and applications: A tutorial," Proc. IEEE, vol. 78, pp. 56-93, Jan. 1990.

[10] T. Q. Nguyen and P. P. Vaidyanathan, "Maximally decimated perfectreconstruction FIR filter banks with pairwise mirror-image analysis and synthesis frequency responses," IEEE Trans. Acoust., Speech, Signal Processing, vol. 36, pp. 693-706, May 1988.

[11] M. J. Smith and T. P. Barnwell, III, "Exact reconstruction techniques for tree-structured subband coders," IEEE Trans. Acoust., Speech, Signal Processing, vol. ASSP-34, pp. 434-441, 1986.

[12] M. Vetterli, "A theory of multirate filter banks," IEEE Trans. Acoust., Speech, Signal Processing, vol. ASSP-35, pp. 356-372, Mar. 1987.

[13] M. Vetterli and C. Herley, "Wavelets and filter banks: Theory and design," IEEE Trans. Acoust., Speech, Signal Processing, vol. 40, pp. 2207-2232, Sept. 1992.

[14] G. Strang and T. Nguyen, Wavelets and Filter Banks. Wellesley, MA: Wellesley-Cambridge, 1996.

[15] R. K. Young, Wavelet Theory and Its Applications. Boston, MA: Kluwer, 1993. 
[16] L. G. Weiss, "Wavelets and wideband correlation processing," IEEE Signal Processing Mag., vol. 11, pp. 13-32, Jan. 1994.

[17] D. C. Schleher, MTI and Pulsed Doppler Radar. Norwood, MA: Artech House, 1991.

[18] J.-M. Lina and M. Mayrand, "Parameterizations for complex daubechies wavelets," in Proc. SPIE Wavelet Applicat. Conf., Orlando, FL, Apr. 1994, vol. 2242, p. 868.

[19] H.-P. Pan, "General stereo image matching using symmetric complex wavelets," in Proc. SPIE Wavelet Applicat. Signal Image Process. IV, Denver, CO, Aug. 1996, vol. 2825.

[20] J. Magarey and N. Kingsbury, "Motion estimation using complex wavelets," in Proc. Int. Conf. Acoust., Speech, Signal Process., May 1996.

[21] A. V. Oppenheim and R. W. Schafer, Discrete-Time Signal Processing. Englewood Cliffs, NJ: Prentice-Hall, 1989.

[22] I. Daubechies, Ten Lectures on Wavelets. Philadelphia, PA: SIAM, 1992.

[23] _ "Orthonormal bases of compactly supported wavelets," Commun. Pure Appl. Math., vol. XLI, no. 41, pp. 909-996, Nov. 1988.

[24] J.-M. Lina, "Complex daubechies wavelets: Filter design and applications," in Proc. ISAAC Conf., Univ. Delaware, Newark, June 1997.

[25] R. A. Gopinath, "Wavelet and filter banks-New results and applications," Ph.D. dissertation, Rice Univ., Houston, TX, 1993.

[26] P. Steffen, P. N. Heller, R. A. Gopinath, and C. S. Burrus, "Theory of regular m-band wavelet bases," IEEE Trans. Signal Processing, vol. 41, pp. 3497-3511, Dec. 1993.

[27] X.-P. Zhang, "Research on wavelet theory and applications in signal processing," Ph.D. dissertation, Tsinghua Univ., Beijing, China, 1996.

[28] A. H. Tewfik, F. Ainha, and P. Jorgensen, "On the optimal choice of wavelet for signal representation," IEEE Trans. Inform. Theory, vol. 38, pp. 747-765, Mar. 1992.

[29] R. A. Gopinath, J. E. Odegard, and C. S. Burrus, "Optimal wavelet representation of signals and the wavelet sampling theorem," IEEE Trans. Circuits Syst. II, vol. 41, pp. 262-277, Apr. 1994.

[30] J. E. Dennis and R. B. Schnabel, Numerical Methods for Unconstrained Optimization and Nonlinear Equations, First ed. Englewood Cliffs, NJ: Prentice-Hall, 1983.

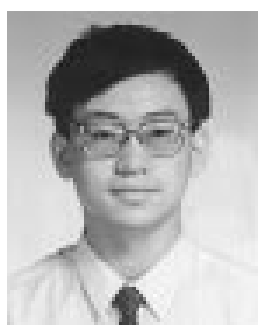

Xiao-Ping Zhang (M'97) was born in 1972 in Xinjiang, China. He received the B.S., M.S., and Ph.D. degrees from Tsinghua University, Beijing, China, in 1992, 1993, and 1996, respectively, all in electronic engineering.

He was a Research Associate with the HighSpeed Signal Processing Laboratory and VHSIC Laboratory, Tsinghua University, from 1990 to 1996. From 1996 to 1998 , he was a Postdoctoral Fellow at the University of Texas, San Antonio, and then at the Beckman Institute, University of Illinois, Urbana-Champaign. He is currently with the Communication Research Laboratory, McMaster University, Hamilton Ont., Canada. His recent research interests include wavelet and filter banks with applications in signal processing, image processing, and communications.

Dr. Zhang received Mei Yiqi fellowship in 1996 from Tsinghua University. $\mathrm{He}$ is also a recipient of the Science and Technology Progress Award from the State Education Commission of China for his contribution to a National High-Tech Project in 1994.
Mita D. Desai (SM'94), photograph and biography not available at the time of publication.

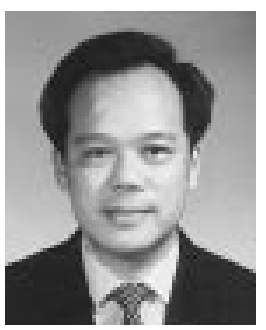

Ying-Ning Peng (SM'96) received the B.S. and postgraduate degrees in electrical engineering from the Tsinghua University, Beijing, China, in 1962 and 1965 , respectively.

Since 1965, he has been with the Department of Electronic Engineering at Tsinghua University, where he is currently a Full Professor and the Director of the Division of Signal Detection and Processing. He has worked with high-speed and real-time signal processing for many years. His recent research interests include spectral estimation, adaptive filtering, adaptive array signal processing, and parallel signal processing.

Prof. Peng has received many awards for his contributions to research and education in China. 